\title{
Are the Electrospray Mass Spectra of Proteins Related to Their Aqueous Solution Chemistry?
}

\author{
Roger Guevremont, K. W. M. Siu, J. C. Y. Le Blanc*, and S. S. Berman \\ Institute for Environmental Chemistry, National Research Council of Canada, Ottawa, Ontario, Canada
}

The shape of the profile described by the relative abundances of multiply charged ions of
proteins in the electrospray mass spectrum can be described by means of one or more
Gaussian functions. An aqueous solution equilibrium model of the distribution of multiply
charged ions of equine cytochrome $c$ and myoglobin has been shown to match qualitatively
the shape of the distribution of these ions in an electrospray mass spectrum. Monotonic
functions such as the quadrupole mass spectrometric transmission effeciency may alter the
centroid of the profile, but the shape of the ion abundance pattern appears to be controlled
by the aqueous solution chemistry of the proteins. (f Am Soc Mass Spectrom 1992, 3, 216-224)

$\mathrm{I}$

n the late 1960s, Dole and co-workers $[1,2]$ described the formation of gas-phase ions when a liquid was sprayed out of a capillary held at high voltage, into a zone of high electrical field. Since that time, the efforts of Fenn and co-workers [3-6] in this research area have led to the development of electrospray mass spectrometry. Instrumentation for electrospray quadrupole mass spectrometry is now commercially available as a result of the work of Iribarne, Thompson, and their colleague [7-9], Covey et al. $[10]$, and Fenn and co-workers $[6,11]$. More recently, methods of introduction of electrospray ions have been developed for sector mass spectrometers [12-14].

Electrospray ionization holds considerable potential for measurement of the molecular masses of large biological molecules $[6,10,11]$. In addition, recent work by Kebarle and co-workers $[15,16]$ suggests that, in the future, significant new activities in the areas of organic and inorganic electrospray mass spectrometry will develop.

Most of the reports dealing with electrospray have suggested mechanisms of ion formation. It is generally believed that the ions exist, preformed in solution, and are subsequently "evaporated" into the gas phase. This suggests, therefore, that the mass spectrum should reflect the aqueous solution chemistry within the droplet as ion desorption proceeds. This has been further supported by recent reports showing that the electrospray spectrum reflects the conformation of proteins in solution [17-19]. The difficulty in

\footnotetext{
NRCC No. 32938

* Postgraduate student, Department of Chemistry, Queen's University, Kingston, Ontario, Canada K7L 3N6.

Address reprint requests to Roger Guevremont, Institute for Environmental Chemistry, National Research Council of Canada, Montreal Road, Ottawa, Ontario, Canada K1A OR9.
}

achieving a quantitative comparison between the electrospray spectrum and the solution chemistry is that the specific chemical state of the droplet is unknown during the critical moment of ion formation $[7,8,11$, 20].

In this report, we take the position that, although the exact aqueous solution state of the droplet $(\mathrm{pH}$, temperature, solvent composition, etc.) is unknown, it is possible to compare the distribution of the ions of protein in aqueous solution qualitatively with the series of multiply charged ions observed in the electrospray mass spectrum. A protein exists in solution in a distribution of charge states. This distribution depends on solution properties, particularly $\mathrm{pH}$. Furthermore, this distribution depends on the pKa's of the acidic and basic amino acids in the protein. The question we pose in this report is whether, despite the changes in the aqueous solution during formation of droplets, the ion abundance distribution in the electrospray spectrum resembles the distribution of preformed multiply charged protein ions in the bulk aqueous solution. A more extensive comparison should be possible in the future, assuming that a detailed model of the solution chemistry of the droplets can be proposed.

We will also consider whether known (or unknown) factors may act in such a way to significantly modify the ion abundance distribution. A function that could modify, but not necessarily disguise, the original ion abundance pattern is the mass-dependent transmission efficiency of the quadrupole mass spectrometer. Factors that may modify the distribution include gas-phase reactions $[15,16]$ or desolvation processes which would lead to a change of mass, charge, or both. The efficiency of electrospray ion formation may be dependent on factors such as the 
charge to mass [11] (or perhaps charge to volume) ratio of the particular ion. Such functions, irrespective of their chemical/physical origin, would mathematically consist of ion "formation efficiency envelopes," and would be superimposed upon the other functions described above.

\section{Experimental}

Electrospray experiments were performed by using a TAGA 6000E (SCIEX Inc., Thornhill, Ontario, Canada) triple quadrupole mass spectrometer. The system has an upper mass range of $m / z 1400$. With the scans appropriate for the present work, the instrument performs measurements at integral mass steps. To ensure reliable measurement of relative ion abundance data in complex spectra, the mass resolution was decreased from unit mass resolution to the extent that two or more measurements were taken near the apex of every peak.

Samples were continuously infused at a rate of 20 $\mu \mathrm{L} / \mathrm{min}$ through a $100-\mu \mathrm{m}$ i.d. stainless steel capillary held at $3.5 \mathrm{kV}$. Stable operation of electrospray was achieved with the needle $-1.5 \mathrm{~cm}$ from the instrument orifice, and with a measured stable current of between 20 and $50 \mathrm{nA}$ flowing between the needle and the system ground. At $\mathrm{pH}$ above 5, and ionic strength below 0.0001 , electrospray signals were enhanced with the electrospray needle located at greater distances from the mass spectrometer orifice.

Data acquisitions considered in this work were run in replicate, and the experiments were then repeated over a duration of 2 months. During this time, the relative abundances of ions within the spectra were observed to be relatively independent of mass spectrometer operating conditions. The spectra collected agree very well with published spectra.

Cytochrome $c$ (horse heart, C-2506 type III) and myoglobin (horse skeletal muscle, M-0630) were obtained from Sigma Chemical Co. (St Louis, MO). Cytochrome $c$ samples $(20 \mu \mathrm{g} / \mathrm{mL}$ ) were prepared to cover various $\mathrm{pH}$ ranges: from $\mathrm{pH} 2.5$ to $\mathrm{pH} 3.6$ with $\mathrm{HCl}$, from $\mathrm{pH} 2.8$ to $\mathrm{pH} 3.5$ with formic acid, and from $\mathrm{pH} 3.5$ to $\mathrm{pH} 3.9$ with acetic acid. Each of these cytochrome $c$ solutions was in turn diluted to yield a series of five samples from $30 \%$ to $55 \%$ methanol by weight.

Similarly, three series of myoglobin solutions (20 $\mu \mathrm{g} / \mathrm{mL}$ ) were prepared: from $\mathrm{pH} 2.9$ to $\mathrm{pH} 3.7$ with $\mathrm{HCl}$, from $\mathrm{pH} 2.7$ to $\mathrm{pH} 3.3$ with formic acid, and from $\mathrm{pH} 3.2$ to $\mathrm{pH} 3.6$ with acetic acid. In turn, each solution was diluted to yield a set of four samples ranging from $30 \%$ to $50 \%$ methanol by weight.

The $\mathrm{pH}$ measurements were taken with a standard glass electrode standardized with aqueous solutions. The $\mathrm{pH}$ measurement error introduced by changes in the liquid junction potential and hydrogen ion activity coefficient when such measurements are taken in a solution of $50 \%$ methanol by weight is $\sim 0.13 \mathrm{pH}$ units [21]. The measurements reported here have not been corrected for this effect.

\section{Results and Discussion}

The electrospray mass spectra of proteins are characterized by a series of multiply charged ions. Assuming that the compound (i.e., electrically neutral) mass is $M$, the mass to charge ratio of these ions is described by $(M+c) / z$, where the charge on the ion is $z=1,2,3, \ldots$, etc., and $c$ is the total mass of the cations (e.g., protons) that impart the total charge $z$ to the ion via cation addition [6]. The mass to charge ratio separation between these ions is not constant and gives the ions an appearance of being more closely spaced at higher charge (i.e., at lower mass to charge ratio). This has the effect of giving the ion relative abundance pattern a slightly skewed appearance. A similar abundance plot using $z$ as the $x$-axis yields a relative abundance profile with a symmetrical distribution.

Proteins exist in an acidic solution as a series of multiply charged protonated ions. In this report we consider whether there is a correlation between the distribution of preformed ions in bulk aqueous solution (for lack of information about the droplet chemistry) and the distribution of multiply charged ions observed in electrospray mass spectra. The following discussion will first deal with the mathematical aspects of the shape of the ion abundance pattern (fitted to a Gaussian distribution), and then attempt to compare that pattern with the aqueous acid-base chemistry of proteins.

\section{Mathematical Analysis of the Electrospray Ion Abundance Pattern (Gaussian Model)}

The electrospray ion abundance pattern $l(z)$, for example, that of cytochrome as shown in Figure 1a, can be described by the normalized Gaussian distribution function:

$$
I(z)=\frac{1}{\sigma \sqrt{2 \pi}} e^{-(1 / 2)\left[(z-\mu)^{2} / \sigma^{2}\right]}
$$

where $z$ is the ionic charge, $\mu$ is the mean charge, and $\sigma$ is the width of the function.

The mean charge $\mu$ on a protein, is a function of $\mathrm{pH}$. Equation 1 may be used to describe the electrospray ion abundance profile throughout the experimental conditions used in this work. The Gaussian profile with $\mu$ of 17.3 matches (least squares $\mathrm{ft}$ ) a cytochrome $c$ spectrum acquired from an $\mathrm{HCl}$ solution of $\mathrm{pH} 3.0$, whereas $\mu$ of 15.6 fits a spectrum acquired from an acetic acid solution at $\mathrm{pH}$ 3.9. At low $\mathrm{pH}$ the width $\sigma$, of the abundance profile, is a constant. Table 1 includes the mean and width parameters, which give good fits of the Gaussian function to the electrospray of cytochrome $c$. These calculations are based on the electrospray spectra shown in Figure 2 (how- 

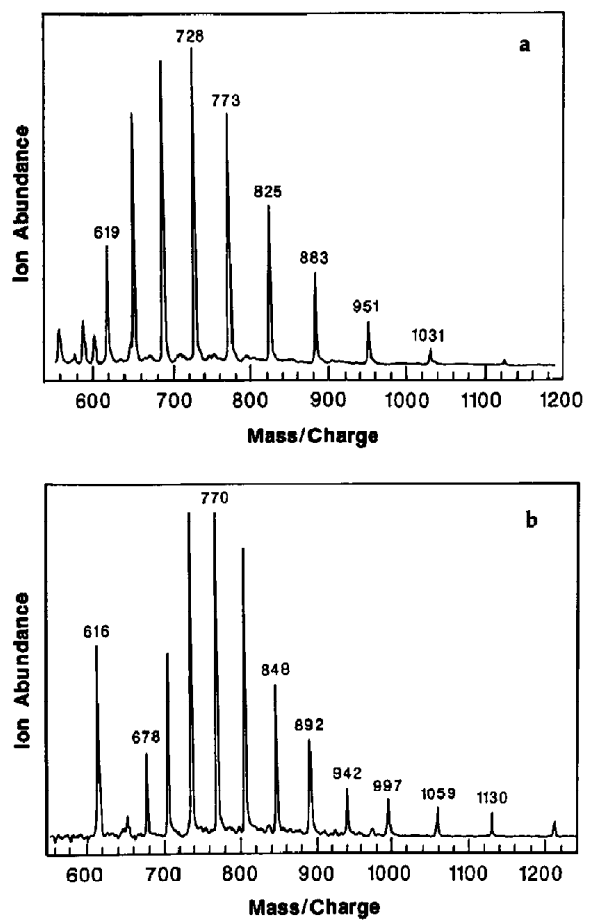

Figure 1. Electrospray mass spectrum of (a) cytochrome $c, \mathrm{pH}$ 3.0 with $\mathrm{HCl}, 50 \% \mathrm{MeOH}$, and (b) of myoglobin, $\mathrm{pH} 2.9$ with $\mathrm{HCl}, 50 \% \mathrm{MeOH}$.

ever, the calculated Gaussian profiles are not shown in this figure).

The ion abundances pattern of myoglobin (Figure $1 \mathrm{~b}$ and Figure 3 ) is somewhat more complex than that of cytochrome $c$. It is apparent that the profile is composed of at least two superimposed Gaussian functions. Previous work with myoglobin has also revealed a shoulder on the high mass to charge ratio side of the profile [22].
Several ion abundance patterns for myoglobin are illustrated in Figure 3 . These profiles were fitted (least squares) to a Gaussian function using parameters summarized in Table 1 (the curves are not shown in Figure 3). Under some experimental conditions the ion abundance profiles can be best described as the sum of two Gaussian functions, each having their own mean charge values $\mu_{a r}$ and $\mu_{b}$, and their own values of $\sigma$. The series of profiles can be described by variation of the values of $\mu$ and the relative weights which the two functions contribute to the profile.

The Gaussian function in this case has a purely descriptive mathematical role: (1) it requires two parameters to describe each experimental spectrum, and (2) it provides no insight into the physical or chemical origin of the electrospray spectrum.

\section{Aqueous Solution Chemistry of Proteins at Low $\mathrm{pH}$}

Cytochrome $c$ has 24 basic amino acids (lysine, arginine, histidine, and amino terminus acetylated), and 15 acidic (carboxylic acid) sites [23], including the carboxyl terminus and the two propionic acids on the heme group (but not including amino acids containing $-\mathrm{CONH}_{2}$ side chains). It is believed that the iron within the heme group is coordinated to a histidine and methionine residue [24]. Since the heme group is covalently linked to the protein through cysteine residues, the heme remains linked to cytochrome $c$ even at low $\mathrm{pH}$ and in the presence of methanol. We will assume that the heme-bound histidine can be protonated within the $\mathrm{pH}$ range considered here; thus the total number of available bases in cytochrome $c$ will be taken to be 24 . It has been suggested that the total charge may be +25 [25] if the oxidation state of the heme iron is taken to be +3 .

In aqueous solution, at the isoelectric point of this protein ( $\mathrm{pH} 10.65$ ), the species has no net electric charge. In other words, all of the acidic groups (15) are negatively charged and an equal number (15) of the basic groups are positively charged.

As the $\mathrm{pH}$ is lowered from the isoelectric point, the protein will pass through two distinct regimes. From

Table 1. Electrospray ion abundances of multiply charged protein ions:

A comparison of aqueous solution and Gaussian distribution models

\begin{tabular}{|c|c|c|c|c|c|c|c|c|c|c|c|c|}
\hline \multirow[b]{2}{*}{ Protein } & \multirow[b]{2}{*}{ Data set } & \multicolumn{2}{|c|}{ Experimental } & \multicolumn{5}{|c|}{ Gaussian model ${ }^{a}$} & \multicolumn{4}{|c|}{ Solution equilibrium mode } \\
\hline & & Acid & $\mathrm{pH}$ & $\mu_{\mathrm{a}^{a}}^{\mathrm{a}}$ & $\sigma_{\mathrm{a}}$ & $\mu_{\mathrm{b}}$ & $\sigma_{\mathrm{b}}$ & $b / a^{b}$ & $\mathrm{pH}$ & $\mathrm{pK}_{\text {int }}{ }_{\mathrm{g}}^{\mathrm{c}}$ & $\mathrm{pK}_{\text {int }_{\mathrm{b}}}$ & $b / a$ \\
\hline \multirow[t]{3}{*}{ Cytochrome $c$} & A & $\mathrm{HCl}$ & 3.0 & 17.3 & 1.99 & - & - & - & 3.10 & 4.0 & - & - \\
\hline & B & acetic & 3.9 & 15.6 & 2.08 & - & - & - & 3.35 & 4.0 & - & - \\
\hline & $\mathrm{C}$ & acetic & 4.7 & 12.1 & 1.3 & - & - & - & 3.90 & 4.0 & - & - \\
\hline \multirow[t]{4}{*}{ Myoglobin } & $\mathrm{D}$ & $\mathrm{HCl}$ & 2.9 & 22.2 & 2.0 & - & - & - & 2.80 & 3.8 & - & - \\
\hline & E & formic & 2.9 & 22.8 & 2.13 & 17.7 & 2.4 & 0.26 & 2.75 & 3.8 & 3.25 & 0.25 \\
\hline & $\mathrm{F}$ & acetic & 3.8 & 21.5 & 2.5 & 16.7 & 2.2 & 0.47 & 2.84 & 3.8 & 3.35 & 0.60 \\
\hline & G & $\mathrm{HCl}$ & 4.0 & 18.5 & 1.5 & 15.3 & 1.1 & 0.47 & 3.22 & 3.8 & - & - \\
\hline
\end{tabular}

${ }^{2} \mu$ and $\sigma$ are the mean charge and width of the Gaussian function, respectively.

$b / a$, weighting factor.

Intrinsic $\mathrm{pK}_{\text {int, }}$ from titration data, $\mathrm{pK}_{\text {int }_{\mathrm{h}^{\prime}}}$ discussed in text. 

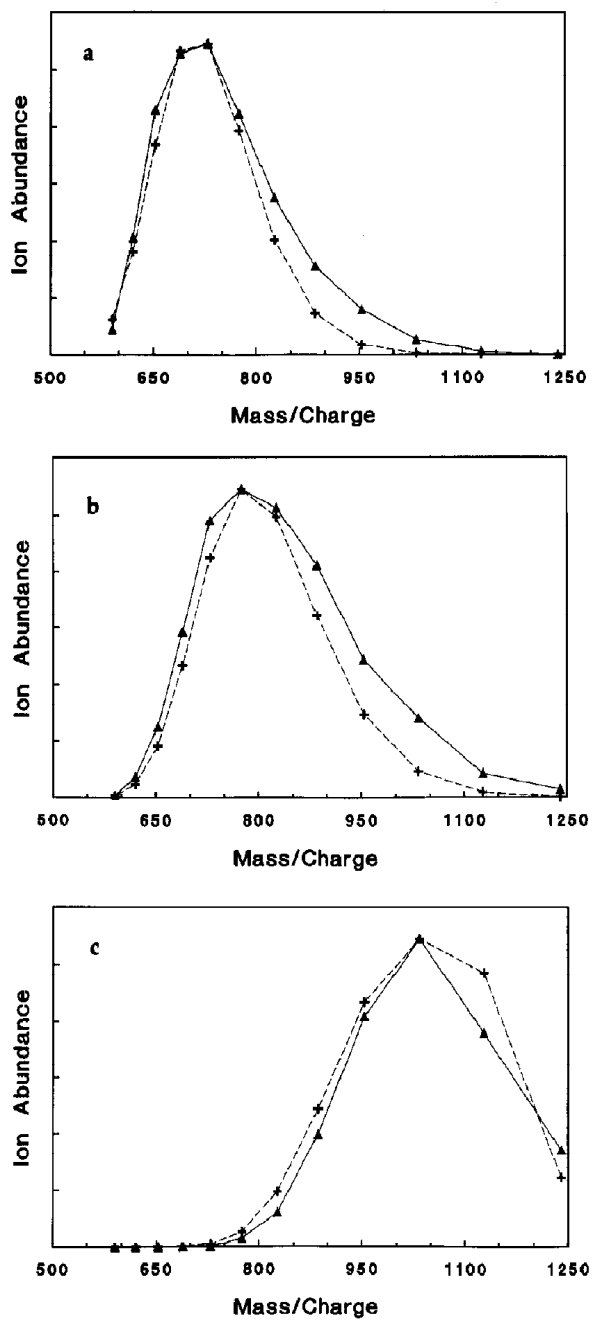

Figure 2. Ion abundance profiles of multiply charged cytochrome $c$ : Experimental conditions corresponding to each data set and the parameters (acid-base solution equilibria) used to calculate theoretical profiles are shown in Table 1 ; plots a, b. and $c$ correspond to data set A, B, and $C$, respectively. (A) Experimental restults; $(+)$ profiles calculated by means of the acid-base equilibrium model.

the isoelectric point to $\mathrm{pH} 5$, all of the net positive charges which the compound acquires will be attachment of protons to basic amino groups. However, in the region of $\mathrm{pH} 4$ to $\mathrm{pH} 5$ the last of the basic amino acids (including histidine) will be protonated $[26,27]$. At this point all of the acidic groups will be anionic (contributing -15 charges to the protein), and all of the basic groups will be protonated and positively charged (contributing +24 charges). The protein will have a net charge of +9 . This is not the maximum charge that cytochrome $c$ can acquire.

The second regime occurs below $\mathrm{pH} 5$. In this $\mathrm{pH}$ region the addition of protons to the carboxylic acids controls the net charge on the species in aqueous solution. For example, all of the basic groups of cytochrome $c$ are protonated ( +24 charges) and any further increase in the net positive charge of the aqueous species requires protonation of some of the 15 acidic groups (i.e., neutralization of the negative $\mathrm{COO}^{-}$groups). At $\mathrm{pH}$ below 1.5 (if the protein does not undergo primary structure degradation) the last of the carboxylic acids may be protonated, and the compound could in theory reach the ultimate +24 (or +25 ) net charges.

In considering the aqueous solution chemistry of these compounds below pH 5 , we may, therefore, disregard the basic amino acids, and concern ourselves only with the protonation of the carboxylic acid groups. The addition of the first proton to a carboxylic acid group in cytochrome $c$ will be less favorable than the addition of a proton to acetic acid (pKa 4.75) because the protein already possesses a net charge of +9. The pKas of the side chain carboxylic acid groups of aspartic and glutamic acids are 3.86 and 4.25 , respectively. The pKas of the carboxylic group of lysine, histidine, and arginine (pKa 2.18, 1.82, and 2.17, respectively) may be guides to the approximate acidity of the most acidic protons on small acids which have a net charge of +1 .

The protonation of lysine (pKa 2.18) at low $\mathrm{pH}$ ( $z=+1$ to $z=+2$ ) is illustrated as follows:<smiles>[NH3+]CCCCCCCCCCCCCC(=O)[OH2+]</smiles>

Note also that, at a $\mathrm{pH}$ equal to the $\mathrm{pKa}(2.18)$, the two charged species of lysine will coexist in solution at a 1:1 ratio. The distribution of coexisting, multiply charged ions of protein in aqueous solution is an extrapolation of this very simple case.

\section{Aqueous Solution Equilibrium Model for the Ion Abundance Pattern of Cytochrome $\mathrm{c}$}

The electrostatic repulsion between a charged protein and an approaching proton reduces the pKas of any remaining acidic groups. The magnitude of this decrease has been estimated [28-30]. The activity coefficient for a charged species is given by:

$$
\ln \gamma=z^{2} w
$$



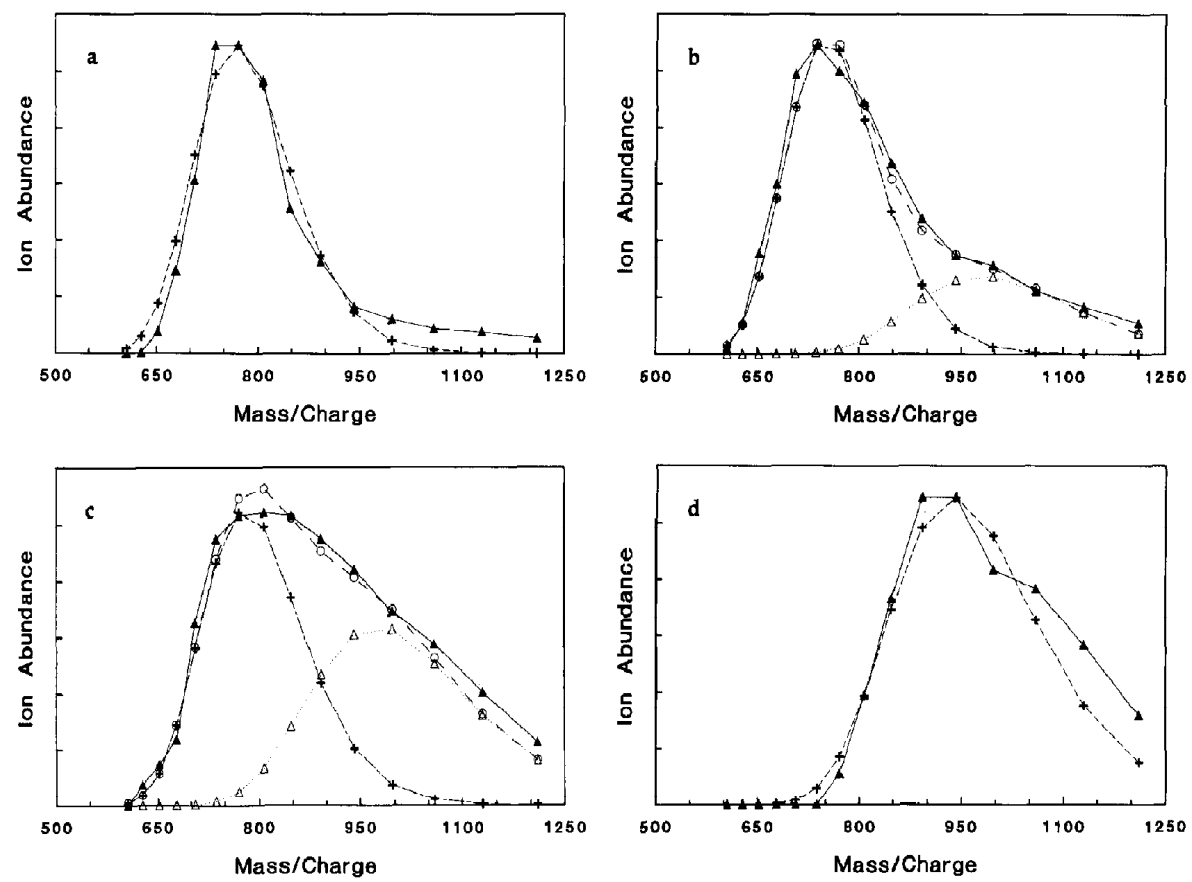

Figure 3. Ion abundances profles of multiply charged myoglobin: Experimental conditions corresponding to each data set and the parameters (acid-base solution equilibria) used to calculate theoretical profiles are shown in Table 1; plots a, b, $\mathbf{c}$, and $\mathbf{d}$ correspond to data set $D, E, F$, and $G$, respectively. ( $\Lambda$ ) Experimental results; $(+)$ profiles calculated using the acid-base equilibrium model and $\mathrm{pK}_{\text {int }}$ of $3.8 ;(\triangle)$ additional theoretical profiles using $\mathrm{pK}_{\text {int }}$ and weighting as shown in Table $1 ;(0)$ sum of profiles.

where $\gamma$ is the activity coefficient, $z$ is the charge on the species, and $w$ is a term related to the dimensions of the ions, the dielectric constant of the medium, and to the ionic strength of the solution. The $\mathrm{pKa}$ of multiply charged species may be adjusted using these activity coefficients to yield:

$$
K_{H_{n} A}=\frac{\left[H_{(n-1)} A\right][H]}{\left[H_{n} A\right]} \frac{e^{(z-1)^{2} w}}{e^{z^{2} w}}
$$

From eq 3, it is apparent that the size of the correction term is a function of the charge on the species. After rearrangement of eq 3 , it is possible to show that the (electrostatic only) change in pKa with each increase of one positive charge is:

$$
\Delta p K_{a}=\frac{-2 w}{2.303}
$$

In a more extensive treatment of the acid-base chemistry of the protein as a polycarboxylic species, the acid dissociation of the $x$ th carboxylic site may be described [29] by

$p K_{x}=p K_{0}^{0}-0.868 w(n-x+0.5)-\log \left(\frac{m-x+1}{x}\right)$

wherein

$$
K_{0}^{0}=K_{0} \cdot e^{ \pm b}, \quad w=b\left[1-\frac{\kappa r}{1+\kappa a}\right], \quad b=\frac{e^{2}}{2 \epsilon k T}
$$

and $m$ is the number of carboxyl groups, $m$ is the number of basic sites, $a$ is the distance of closest approach of the ions, $b$ is the charge interaction term of Bjerrum [28], $r$ is the distance separating the proton from the charge of the protein in the theory of Bjerrum, and $\kappa$ is the function of ionic strength in the Debye-Hückle theory. The intrinsic acid dissociation constants $\left(\mathrm{pK}_{0}\right)$ of aspartic acid and glutamic acids are $\sim 4.0$ and 4.5 , respectively [31].

From $\mathrm{pH}$ titration data [25, 32-34], the acid dissociation constants of cytochrome $c$ can be estimated by using the methods described above. More complex models have recently been described $[35,36]$. 
The titration data reported for cytochrome $c$ [25, 33, 34] show that the rate of change of the charge $(\mathrm{z})$ on the protein is $\sim 6.5$ per $\mathrm{pH}$ unit from below $\mathrm{pH} 3$ to over $\mathrm{pH} 4$ (linear within this $\mathrm{pH}$ range). The data also show that the net charge on the protein is $\sim+11$ at $\mathrm{pH} 4$ and +17 at $\mathrm{pH} 3$. Given that cytochrome $c$ contains 13 carboxylic sites (Asp and Glu), and that the intrinsic pKas of the two heme propionic sites are 4.8 and 5.4, respectively [33], it is evident (based on titration data between $\mathrm{pH} 3$ and $\mathrm{pH}$ 4) that the first carboxylic group to be protonated has a pKa of 4.0 $\left(\mathrm{pK}_{\text {int }}\right)$ and that each subsequent group has a pKa of $\sim 0.15$ unit lower (this decrease is a linear series within the $\mathrm{pH}$ range considered here). The pKas of the 13 (Glu and Asp) sites are therefore 4.0, 3.85, 3.7, etc., with the most acidic site having a pKa of $\sim 2$.

The treatment of Linderstrom-Lang, eq 5 above, and the titration data below $\mathrm{pH} 3$, suggest that the pKas of the most acidic sites are considerably (at least 0.5 units) lower than the estimate described above. However, because of the limited $\mathrm{pH}$ range used during acquisition of the electrospray spectra described in this report, and because of the inherent limitations in the accuracy of $\mathrm{pH}$ titrations below $\mathrm{pH} \mathrm{3}$, a more accurate treatment at very low $\mathrm{pH}$ is not warranted in this report.

The distribution of the multiply charged ions of cytochrome $c$ can be calculated using the pKas of the carboxylic acid groups and the bulk solution $\mathrm{pH}$. Figure 2 illustrates a comparison of the shape of the experimental electrospray ion abundance pattern, and the distribution of multiply charged protein ions calculated on the basis of the pKas of the carboxylic groups determined from titration data as described above. The $\mathrm{pH}$ of the solutions used for acquisition of the electrospray spectra and the $\mathrm{pH}$ values used to calculate the distributions appear in Table 1 . The discrepancy between the experimental (electrospray) $\mathrm{pH}$ and the $\mathrm{pH}$ used to calculate the distribution could be a result of the shift of the distribution by other processes at work during the electrospray experiment, including evaporation of the solvent and/or volatile acids. The effect of one function, namely the ion transmission efficiency through the quadrupole mass spectrometer is considered below. Such functions may shift the distribution to appear as if it originated from a solution at a $\mathrm{pH}$ different from that experimentally determined for the bulk aqueous solution. However, it is important to note that although the position of the centroid cannot be quantitatively calculated at this time, the shape and width of the distribution will not be significantly modified by such functions.

\section{Aqueous Solution Equilibrium Model for the Ion Abundance Pattern of Myoglobin}

Myoglobin (equine skeletal muscle) has 23 acidic groups including the carboxyl terminus and the heme group (excluding amino acids containing $-\mathrm{CONH}_{2}$ side chains), and 33 basic amino acids including the amino terminus. At neutral $\mathrm{pH}$, it is believed that two histidine residues are involved in heme binding [24]. However, the electrospray spectra reported here have been acquired under conditions (low $\mathrm{pH}$, aqueous methanol mixtures) wherein the labile heme has separated (note the presence of $m / z 616$ in Figure $1 \mathrm{~b}$ ) from the protein, and the maximum charge which the protein can acquire will therefore be +33 .

The ion abundance pattern of myoglobin is more complex than that of cytochrome $c$ in that under some experimental conditions it appears to be a sum of two distribution functions. (Cytochrome $c$ will also exhibit this dual function distribution in solutions containing little methanol and/or acid, but not under the experimental conditions employed in this report.) Bimodal ion abundance distributions have been attributed to changes in the conformation of the protein in solution [17-19].

The equilibrium model for the ion abundance pattern of myoglobin is constructed in exactly the same manner as that for cytochrome $c$. The $\mathrm{pH}$ titration data for sperm whale myoglobin $[34,37,38]$ shows that the linear rate of change of net charge $(\mathrm{z})$ on the protein is -9.5 per $\mathrm{pH}$ unit within the range from $\mathrm{pH} 3$ to $\mathrm{pH} 4$. This indicates that the pKas of the carboxylic groups are separated by $\sim 0.105$ units (linear in the appropriate $\mathrm{pH}$ range). The net charge on the horse muscle myoglobin is expected to be -+11 at $\mathrm{pH} 4$ and +20 at $\mathrm{pH} 3$. This match to the titration data is possible if the pKa of the first carboxylic group to be protonated is taken to be 3.8 . The subsequent pKas are therefore $\sim 3.7,3.6,3.5$, etc. Note that the pK $_{\text {int }}$ is lower than that reported by Nozaki and Tanford [31], and lower than that observed for $\mathrm{cy}$ tochrome $c$ as described in the previous section. This difference, however, is not significant. The intrinsic pKas reported by Marini and Martin [39] were 3.8 for glutamic acid and 3.0 for aspartic acid, in a model of the titration of cytochrome $c$ in which the electrostatic repulsion factor $w$ (eq 2) was determined to be zero.

Figure 3 illustrates the comparison of the distribution of multiply charged ions in the electrospray spectra of myoglobin, and the distribution of preformed protein ions in the bulk solution. Table 1 summarizes the experimental and calculation parameters used for this figure. Under some conditions the experimental ion abundance profiles for the electrospray mass spectrum of myoglobin cannot be matched with a single set of pKas calculated in the manner described above. These electrospray results suggest that the data are a superimposition of two distribution functions. Figure $3, b$ and $c$, illustrates this situation. For illustrative purposes, the two theoretical profiles are calculated based upon differing $\mathrm{pK}_{\mathrm{int}}$ values, one set based on an intrinsic pKa of 3.8, and the second based on an intrinsic pKa of $\sim 3.3$. In all other regards (aqueous solution $\mathrm{pH}$ and change of pKa per carboxylic site) the models are identical. It should be pointed out that the 
second $\mathrm{pK}_{\mathrm{int}}$ values were not calculated from the $\mathrm{pH}$ titration data. However, titration data [37] strongly suggest that myoglobin exists in more than one conformation in solution, particularly from $\mathrm{pH} 4$ to $\mathrm{pH} 5$ wherein denaturation of the protein is apparent.

We note here that, from a mathematical point of view, two possible explanations of the overlapped distributions noted above for myoglobin should be considered. These are experimentally indistinguishable at this point. First, the solution may be composed of two forms of myoglobin, but in different three-dimensional configurations with one being more compact than the other. The result would be a set of unique pKa values for each configuration since the electrostatic environment of the states is substantially different. The mass spectra would simply reveal the sum of the two distributions. Second, myoglobin may possess two sets of carboxylic acid moieties with clearly different properties. From a strictly mathematical point of view, the observed data can be explained by two series of pKas which differ only in the spacing between the pKas of consecutive dissociations. If the aqueous solution $\mathrm{pH}$ is similar to the $\mathrm{pKa}$ where the transition between the two series occurs, then nonsymmetrical profiles such as seen in Figure 3, b and c, will be observed.

\section{Mass Spectrometer Ion Transmission Function}

The ion transmission efficiency of a quadrupole mass spectrometer is a function of mass. The transmission roll-off of a SCIEX TAGA instrument of this type has been estimated [40] to be over an order of magnitude per $200 \mathrm{~m} / \mathrm{z}$ units.

Because of the strong bias of the mass spectrometer transmission function, it is reasonable to expect that distortion of the relative abundances of multiply charged ions in an electrospray spectrum could occur, especially since these distributions often span several hundred mass to charge units. Bias of this type will make it difficult to quantitatively match the centroid of ion abundance profile observed in the spectrum, to the centroid of the abundances of preformed multiply charged ions in aqueous solution. The effect of the mass spectrometer transmission efficiency function is to shift the apparent centroid of the ion distributions toward lower mass.

Figure 4 illustrates the ion abundance profile for the set of experimental data for cytochrome $c$ (pH 3.0, $\mathrm{HCl}$ ) shown in Figure 2a. The $\mathrm{pH}$ used to calculate the theoretical ion abundances in Figure 2a was 3.10, but in Figure 4 the ion abundances were calculated

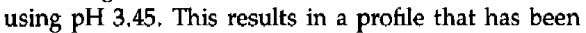
shifted to higher mass-to-charge ratio values. However, if a mass spectrometer roll-off function of a factor of 60 per $200 \mathrm{~m} / \mathrm{z}$ units (measured [40]) is used to modify this calculated ion abundance profile, the resulting profile will shift to a lower mass to charge

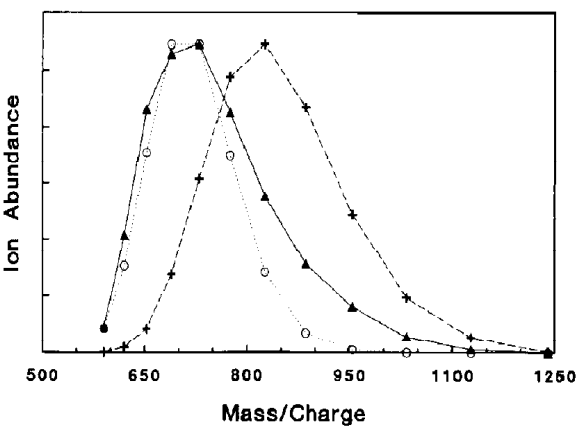

Figure 4. Effect of ion transmission efficiency on the abundance profile of cytochrome $c$ : (A) Experimental results (data set A, Table 1$) ;(+)$ calculated profile at $\mathrm{pH} 3.45 ;(0)$ calculated profile at $\mathrm{pH} 3.45$ with a transmission roll-off of a factor of 60 per $200 \mathrm{~m} / \mathrm{z}$ units.

ratio (as shown in Figure 4), and once again match the experimental data in the manner shown in Figure 2a.

Since all of the ions shown in Figure 4 have massto-charge ratios higher than 500 , the mass spectrometer ion transmission efficiency function is monotonic decreasing at an approximately exponential rate. Under these conditions the centroid of the ion abundance profule is altered, but the fundamental shape of the ion distribution remains unchanged. From a mathematical point of view, the transmission roll-off modifies the ion abundance profile, but in such a way that the new profile may be described by a change in parameters in either the equilibrium model or the Gaussian distribution model. The existence of mass spectrometer transmission roll-off, therefore, does not contradict or conflict with the hypothesis that the basic shape of the ion abundance pattern is controlled by the aqueous solution chemistry of the proteins.

\section{Mathematical Consequences of the Efficiency of Ion Formation in Electrospray}

The efficiency of the formation (e.g., ion evaporation from solution) of a particular ion, in electrospray, may be dependent on such factors as the magnitude of the charge to mass [11] (or perhaps charge to volume) ratio of the ion. This would introduce a bias favoring formation of a series of ions which had the appropriate characteristics, and inhibit formation of ions which fall outside of this "most favored" set of parameters. Such a function would mathematically be described as an ion "formation efficiency envelope," which would be superimposed upon the other functions described above (acid-base chemistry, spectrometer transmission roll-off, etc.). What impact would this "envelope" have on the observed electrospray spectrum? The shape of the ion abundance pattern in the electrospray spectrum would only be controlled by such an envelope if the width of the envelope (distribution of 
ions favored by the envelope) is approximately equal to, or narrower than, the distribution of multiply charged ions controlled by acid-base chemistry. If the ion formation efficiency envelope is wide, then the measured ion distribution in the electrospray spectrum will be controlled by the narrowest function, namely the acid-base controlled ion distribution pattern. If the experimental conditions are modified to an extent that there is a mismatch between the ions existing in solution, and the ions favored by the envelope, the abundance of electrospray ions (and the total ion current) will decrease. The shape (and width) of the spectrum will be controlled by the narrowest active distribution function. The distribution of ions experimentally observed in the electrospray spectra appear to be similar in width to the distribution calculated by bulk solution acid-base chemistry.

\section{Aqueous Solution Chemistry of the Droplet during Electrospray and Evaporation}

The electrospray spectra of proteins are usually more easily acquired in mixtures of methanol and water than in aqueous solution alone. The acid-base chemistry of proteins in water-methanol mixtures will deviate from that in pure water. It is also anticipated that the conformational changes of proteins in these mixtures will deviate considerably from that water. The acid-base chemistry of some acids in water-methanol mixtures have been considered [41, 42]. A detailed evaluation of the protein ion distribution in the presence of methanol must be considered in the future, but at this time is beyond the scope of this work.

During the electrospray process, the aqueous solution undergoes many changes in the zone near the probe tip. These changes can alter the concentration of components in the solution. First, the high electrical field at the capillary tip will induce ion migration during the time which the solution is in electrical contact with the probe or with the conductive solution inside it. The hydrated proton, with very high mobility in solution [43], may migrate to an extent that significantly changes the composition of the droplet. As well, protons will be formed via the electrolysis of water, and will act to carry some of the electrical current from the probe into the bulk aqueous solution within the probe (as long as a high enough potential difference exists between a portion of liquid inside the capillary and the capillary wall itself). These effects will be most significant near the tip of the capillary, wherein the solution is influenced by the high electric field, yet also remains in electrical contact with the capillary and/or the solution within the capillary. This flow of protons translated into a decrease in the apparent $\mathrm{pH}$ of the droplets.

Second, the aqueous solution chemistry will change during droplet evaporation. A volatile organic solvent, if present, will evaporate preferentially, with cooling of the droplet. The solvent will evaporate from the droplet, increasing the concentration of electrolytes. Furthermore, volatile acids such as acetic and formic acid will evaporate. The final droplet $\mathrm{pH}$ will depend on the quantity and nature of the acid used to adjust the $\mathrm{pH}$ of the bulk solution.

Third, the droplet will not be homogeneous. Since the droplet will possess an electric charge, the electrolytes in the droplet will migrate in such a way to distribute the excess charge in the most electrostatically favorable manner, probably at the droplet surface. In the case of positively charged droplets, this will probably mean that hydrated protons, and surface-active cations, will preferentially migrate to the droplet surface. Ion desorption and/or Rayleigh division may then be a mechanism or mechanisms whereby the droplet can alleviate the charge built up $[11,44]$.

The processes described above will alter the aqueous solution chemistry of the droplet, and particularly the droplet surface, in such a way that it may be significantly different from that of the original bulk solution. Monotonic biases such as the mass spectrometer transmission function, and others that remain to be identified, skew the mass spectrum but do not change the global picture. The purpose of the present discussion is to consider the physical and/or chemical processes that control the shape of the ion abundance profile in electrospray mass spectrometry, rather than to predict quantitatively the ion abundances from measured physical parameters. These processes appear to originate from the aqueous solution chemistry of proteins.

\section{Conclusions}

The ion abundance profiles observed in the electrospray mass spectrum of proteins can be described by one or more superimposed Gaussian distribution functions. Such functions describe many common physical phenomena. We suggest that the ion abundance profiles may reflect the abundances of preformed, multiply charged species in aqueous solution. Because other functions, including the transmission decay of a quadrupole mass analyzer, can also be described as exponential functions, superimposition of these functions serves to modify the mean and width of the ion abundance pattern, but does not change its basic mathematical description. For example, if the ion abundance pattern is controlled by the aqueous solution chemistry, the quadrupole roll-off function will modify the apparent position of the function on the mass-to-charge ratio scale, but not the shape of the function itself.

The aqueous solution chemistry model for the ion abundance pattern is attractive because it provides a simple physical model for the observed data. The model must pass several tests for consistency. It inust first agree with known solution chemistry of the proteins. The ion abundance profiles of more than one 
protein, over a range of experimental conditions must be described with a consistent set of parameters. Known functions that must be superimposed on the aqueous solution chemistry model, including the quadrupole ion transmission function, should not result in predictions inconsistent with the observed mass spectra. These conditions have all been met by the aqueous solution model.

Many processes contribute to the ion abundance profiles in electrospray mass spectrometry of proteins. We list only a few possibilities: aqueous solution chemistry, kinetics of ion desorption, efficiencies of gas phase ion formation, ion transmission into the vacuum, desolvation and declustering, and transmission efficiencies of electrostatic optics and quadrupole mass filters. We suggest in the present discussion that the fundamental origin of the ion abundance profile observed in electrospray is controlled by the concentration of preformed ions in aqueous solution. If the other functions modify this original pattern, either by exponential monotonic increasing or decreasing functions, or with wide "ion formation efficiency envelopes," then the final ion abundance pattern observed in the mass spectrum will retain the original abundance pattern imposed by aqueous solution chemistry. The distribution of preformed ions in aqueous solution appears to be the most important factor determining ion abundance pattern in the electrospray mass spectrometry of proteins.

\section{References}

1. Dole, M.; Mach, L. L.; Hines, R. L.; Mobley, R. C.; Ferguson, L. P.; Alice, M. B. J. Chem. Phys. 1968, 49, 2240.

2. Mach, L. L.; Kralik, P.; Rheude, A.; Dole, M. I. Chem. Phys. 1970, 52, 4977.

3. Whitehouse, C. M.; Dreyer, R. N.; Yamashita, M.; Fenn, J. B. Anal. Chem. 1985, 57, 675.

4. Meng, C. K.; Mann, M.; Fenn, J. B. Z. Phys. 1988, D10, 361.

5. Wong, S. F.; Meng, C. K.; Fenn, J. B. J. Phys. Chem. 1988, 92,546

6. Mann, M.; Meng, C. K.; Fenn, J. B. Anal. Chem. 1989, 61, 1702.

7. Iribarne, J. V.; Thompson, B. A. J. Chem, Phys. 1976, 64, 2287.

8. Thompson, B. A.; Iribarne, J. V. J. Chem, Phys. 1979, 71, 4451.

9. Thompson, B. A.; Iribarne, J. V.; Dziedzic, P. J. Anal. Chem. 1982, 54, 2219.

10. Covey, T. R.; Bonner, R. F.; Shushan, B. I.; Henion, I. D Rapid Commun. Mass Spectrom. 1988, 2, 249.

11. Fenn, J. B.; Mann, M.; Meng, C. K. ; Wong, S. F.; Whitehouse, C. M. Mass Spectrom. Rev, 1990, 9, 37.
12. Allen, M. H.; Lewis, I. A. S. Rapia Commun. Mass Spectrom. 1989, 3, 255.

13. Meng, C. K.; McEwen, C. N.; Larsen, B. S. Rapid Commun. Mass Spectrom. 1990, 4, 147.

14. Meng, C. K.; McEwen C. N.; Larsen, B. S. Rapid Commun. Mass Spectrom. 1990, 4, 151

15. Blades, A. T.; Jayaweera, P.; Ikonomou, M. G.; Kebarle, P. J. Chem. Phys. 1990, 92, 5900.

16. Jayaweera, P.; Blades, A. T.; Ikonomou, M. G.; Kebarle, P. I. Am. Chem. Soc. 1990, 112, 2452.

17. Chowdhury, S. K.; Katta, V.; Chait, B.T J. Am. Chemt. Soc. 1990, 112, 9012

18. Katta, V; Chait, B. T. Rapid Commun. Mass Spectrom. 1991, $5,214$.

19. Loo, J. A.; Loo, R. R. O; Udseth, H. R.; Edmonds, C. G.; Smith, R. D. Rapid Commmun. Mass Spectrom. 1991, 5, 101.

20. Smith, R. D.; Loo, J. A.; Edmonds, C. G.; Barinaga, C. J. Udseth, H. R. Anal. Chem. 1990, 62, 882.

21. Laitinen, H. A.; Harris, W. E. Chemical Analysis; Mc-GrawHill: New York, 1975

22. Smith, R. D.; Loo, J. A.; Barinaga, C. J.; Edmonds, C. G. Udseth, H. R. I. Am. Soc. Mass. Spectrom. 1990, 1, 53.

23. Fasman, G. D., Ed, Handbook of Biochemistry and Molecular Biology, 3rd ed,, vol. III; CRC Press: Cleveland, 1975.

24. Stryer, L. Biochemistry, 3rd ed.; W. H. Freeman: New York 1988; p. 1089.

25. Marini, M. A.; Marti, G. E.; Berger, R. L.; Martin, C. J. Biopolymers 1980, 19,885.

26. Cohen, J. S.; Hagenmaier, H.; Pollard, H.; Schechter, A. N J. Mol. Biol. 1972, 71, 513 .

27. Wilbur, D. J.; Allerhand, A. J. Biol. Chem. 1977, 252, 4968.

28. Edsall, I. T.; Wyman, J. Biophysical Chemistry, vol. 1; Academic Press: New York, 1958; p. 512.

29. Cannan, R. K.; Palmer, A. H,; Kibrick, A. C. I. Biol. Chem. 1942, 142, 803.

30. Linderstrom-Lang, K. C. R. Trav. Lab. Carslberg, 1924, $15,7$.

31. Nozaki, Y.; Tanford, C. J. Biol. Chem. 1967, 242, 4731.

32. Bull, H. B.; Breese, K. Biochem. Biophys. Res. Commun. 1966, 24,74 .

33. Shaw, R. W. Hartzell, C. R. Biochemistry 1976, 15, 1909.

34. Matthew, J. B.; Friend, S. H.; Botelho, L. H.; Lehman, L. D.; Hanania, G. I. H.; Gurd, F. R. N. Biochem. Biophys. Res. Commun. 1978, 81, 416.

35. Stigter, D.; Dill, K. A. Biochemistry 1990, 29, 1262.

36. Bashford, D.; Karplus, M. Biochemistry 1990, 29, 10219.

37. Breslow, E; Gurd, F. R. N. J. Biol. Chem. 1962, 237, 371.

38. Shire, S. J.; Hanania, G. I. H.; Gurd, F. R. N. Biochemistry $1974,13,2967$

39. Marini, M. A.; Martin, C. J. Anal. Lett. 1980, 13, 93.

40. Sunner, J.; Nicol, G.; Kebarle, P. Anal, Chem. 1988, 60, 1300.

41. Bates, R. G.; Robinson, R. A. In Chemical Physics of Ionic Solutions; Conway, B. E.; Barradas, R. G., Eds; John Wiley and Sons: New York, 1966; p 211.

42. King. E. J. In Physical Chemistry of Organic Solvent Systems, Covington, A. K, Dickinson, T., Eds; Plenum Press: London, 1973; p 331

43. Moore, W. J. Physical Chemistry, 4th ed.; Prentice-Hall: Englewood Cliffs, NJ, 1972; p 435.

44. Mann, M. Org. Mass Spectrom. 1990, 25, 575. 